\title{
GnRH antagonist multiple dose protocol with oral contraceptive pill pretreatment in poor responders undergoing IVF/ICSI
}

\author{
Chung-Hoon Kim', Rae-Mi You', Hyuk-Jae Kang', Jun-Woo Ahn², Ilkyung Jeon', Ji-Won Lee', Sung-Hoon Kim', \\ Hee-Dong Chae', Byung-Moon Kang' \\ 'Division of Reproductive Endocrinology and Infertility, Department of Obstetrics and Gynecology, Asan Medical Center, University of Ulsan College of \\ Medicine, Seoul; ${ }^{2}$ Division of Reproductive Endocrinology and Infertility, Department of Obstetrics and Gynecology, Ulsan University Hospital, \\ University of Ulsan College of Medicine, Ulsan, Korea
}

Objective: To investigate the effectiveness of GnRH antagonist multiple-dose protocol (MDP) with oral contraceptive pill (OCP) pretreatment in poor responders undergoing IVF/ICSI, compared with GnRH antagonist MDP without OCP pretreatment and GnRH agonist low-dose long protocol (LP).

Methods: A total of 120 poor responders were randomized into three groups according to controlled ovarian stimulation (COS) options; GnRH antagonist MDP after OCP pretreatment (group 1), GnRH antagonist MDP without OCP pretreatment (group 2) or GnRH agonist luteal low-dose LP without OCP pretreatment (group 3). Patients allocated in group 1 were pretreated with OCP for 21days in the cycle preceding COS, and ovarian stimulation using recombinant human FSH (rhFSH) was started 5 days after discontinuation of OCP.

Results: There were no differences in patients' characteristics among three groups. Total dose and days of rhFSH used for COS were significantly higher in group 3 than in group 1 or 2 . The numbers of mature oocytes, fertilized oocytes and grade I, II embryos were significantly lower in group 2 than in group 1 or 3 . There were no significant differences in the clinical pregnancy rate and implantation rate among three groups.

Conclusion: GnRH antagonist MDP with OCP pretreatment is at least as effective as GnRH agonist low-dose LP in poor responders and can benefit the poor responders by reducing the amount and duration of FSH required for follicular maturation.

Keywords: Gonadotropin-releasing hormone; Antagonist; Agonist; Oral Contraceptives; Poor Responders; In vitro fertilization; Intracytoplasmic sperm injection; Human

\section{Introduction}

The management for poor responders with diminished ovarian reserve is still a challenge, although many studies have been performed to seek for a method of efficient ovarian stimulation for infertile wom-

Received: Nov 11, 2011 - Revised: Dec 9, 2011 · Accepted: Dec 12, 2011 Corresponding author: Chung-Hoon Kim

Division of Reproductive Endocrinology and Infertility, Department of Obstetrics and Gynecology, Asan Medical Center, University of Ulsan College of Medicine, 388-1 Pungnap 2-dong, Songpa-gu, Seoul 138-736, Korea

Tel: +82-2-3010-3639 Fax:+82-2-3010-6944 E-mail:chnkim@amc.seoul.kr

This is an Open Access article distributed under the terms of the Creative Commons Attribution Non-Commercial License (http://creativecommons.org/licenses/by-nc/3.0/) which permits unrestricted non-commercial use, distribution, and reproduction in any medium, provided the original work is properly cited. en with reduced ovarian reserve. There have been many studies assessing various controlled ovarian stimulation (COS) regimens to improve the outcome of poor responders undergoing IVF, no particular protocol was demonstrated to be best suited for all such patients [1]. GnRH agonist long protocol (LP) has been a standard COS method since it was introduced in assisted reproduction in the late 1980's to prevent premature LH surge and luteinization [2], and GnRH agonist low-dose regimens was advocated as one of important protocols for poor responders in terms of improvement in COS response and reduced duration and dose of gonadotropin stimulation $[3,4]$. More recently, GnRH antagonists have been administered to poor responders during $\mathrm{COS}$ with inconsistent results [5-9]. Although a limited number of randomized controlled trials compare the efficacy of GnRH an- 
tagonist protocol and the agonist long protocol for COS in poor responders undergoing IVF, a meta-analysis by Griesinger et al. [10] demonstrated that $\mathrm{GnRH}$ antagonist protocol results in significantly more cumulus oocyte complexes (COCs) and similar clinical outcome. COS using $\mathrm{GnRH}$ antagonists is also likely to become one of the treatment options for poor responders.

After the introduction of $\mathrm{GnRH}$ antagonists in COS, oral contraceptive pill (OCP) has been used for cycle scheduling purpose. OCP is effective in allowing prediction of timing events in an IVF cycle regarding scheduling and is frequently used before IVF and may influence IVF outcome [11,12]. In a randomized controlled trial by Kolibianakis et al. [13] and a meta-analysis by Griesinger et al. [14], a significant difference in ongoing pregnancy rates between patients who received OCP pretreatment and those who did not was not found. However, subjects for these studies were not poor responders and the available randomized studies on OCP pretreatment in poor responders undergoing IVF have not been reported. It is thought that OCP pretreatment in $\mathrm{GnRH}$ antagonist cycles can result in different ovarian response to COS and IVF results in poor responders, compared with normal responders.

The present study was performed to investigate the effectiveness of GnRH antagonist multiple-dose protocol (MDP) with OCP pretreatment in poor responders undergoing IVF/ICSI, compared with GnRH antagonist MDP without OCP pretreatment and GnRH agonist lowdose LP.

\section{Methods}

\section{Patient population}

A total of 120 poor responders, aged 28-41 years, who were defined as patients with repeated day 3 levels of FSH $>8.5 \mathrm{mlU} / \mathrm{mL}$ [15], and/or antral follicle count (AFC) $\leq 5[16,17]$, were recruited from a university-based infertility clinic at the Asan Medical Center, Seoul, Korea. The Institutional Review Board of our center approved the study and all patients provided written informed consent. Patients were randomized on the basis of a computer-generated list to three groups according to COS options; GnRH antagonist MDP after OCP pretreatment (group 1), GnRH antagonist MDP without OCP pretreatment (group 2) or $\mathrm{GnRH}$ agonist luteal low-dose long protocol without OCP pretreatment (group 3). The sequence of allocation to the three groups was provided to the investigating physicians and randomization was performed as planned according to the randomization list order. All three groups consisted of 40 cycles initiated corresponding to 40 patients, respectively. A probability by power calculation for sample size determination was $81 \%$.

All patients were in good health with normal hepatic, and renal functions with a body mass index of between 19 and $30 \mathrm{~kg} / \mathrm{m}^{2}$. None of subjects had ever taken any infertility medications (clomiphene and/ or gonadotropins) within the preceding three months. Patients with polycystic ovary syndrome according to Rotterdam definition were excluded from this study. Hormonal profiles (serum levels of basal $\mathrm{FSH}, \mathrm{LH}, \mathrm{E}_{2}$, prolactin, testosterone and antimüllerian hormone) and the number of basal antral follicle in ultrasonogram were measured on the 2 nd or 3 rd day of menstruation in all patients.

\section{Ovarian stimulation protocols}

All patients allocated in group 1 were pretreated identically with Minivlar (ethinyl estradiol $0.03 \mathrm{mg}$ and levonorgestrel $0.15 \mathrm{mg}$; Schering AG, Berlin, Germany) for 21 days in the cycle preceding COS. Five days after OCP discontinuation for group 1 and on cycle day 3 for group 2, ovarian stimulation was commenced using recombinant human FSH (rhFSH, Gonal-F; Merck Serono SA, Geneva, Switzerland) of $225 \mathrm{IU} /$ day after establishing ovarian and uterine quiescence using vaginal ultrasound. The rhFSH dose was adjusted according to ovarian response, every 3-4 days. GnRH antagonist (Cetrotide $0.25 \mathrm{mg}$; Merck Serono SA) was started when the leading follicle reached 14 $\mathrm{mm}$ in average diameter, and was continued daily until the day of hCG administration. A recombinant hCG (rhCG, Ovidrel; Merck Serono SA) of $250 \mu \mathrm{g}$ was injected to induce follicular maturation when one or more follicles reached a mean diameter of $\geq 18 \mathrm{~mm}$. Oocyte retrieval was performed 35 to 36 hours after hCG injection and one to four embryos were transferred into the uterus on the third day after oocyte retrieval. Vaginal progesterone gel (Crinone 8\%, $90 \mathrm{mg}$, Merck Serono SA) once daily was administrated from the day of oocyte retrieval, for luteal support. In group 3, the daily injection of $\mathrm{GnRH}$ agonist (Decapeptyl 0.1 mg; Ferring, Malmö, Sweden) was initiated from the midluteal phase and continued until menses followed by a dose reduction to $0.05 \mathrm{mg}$ daily. $\mathrm{GnRH}$ agonist $0.05 \mathrm{mg}$ daily was continued up to day of hCG administration. Ovarian stimulation using $\mathrm{rhFSH}$, oocyte retrieval and luteal support were performed in the same manner with GnRH antagonist MDP.

The serum level of $\beta$-hCG was measured 11 days after embryo transfer (ET). Measurement of $\beta$-hCG was performed by radioimmunoassay using a hCG MAIAclone kit (Serono Diagnostics, Woking, UK); interassay and intraassay variances were less than $10 \%$ and $5 \%$, respectively.

\section{Outcome measures}

Primary efficacy endpoint was the number of mature oocytes retrieved. Secondary efficacy variables included total amount and days of rhFSH administered, the numbers of fertilized oocytes and grade I, Il embryos, implantation rate, clinical pregnancy rate per cycle, and live birth rate per cycle.

Clinical pregnancy was defined as the presence of a gestational sac 
by ultrasonography 5 weeks after oocyte retrieval, while miscarriage rate per clinical pregnancy was defined as the proportion of patients who failed to continue development before 20 weeks of gestation in all clinical pregnancies. Live birth was defined as the delivery of a fetus with signs of life after twenty four completed weeks of gestational age.

\section{Statistical analysis}

The mean value was expressed as the mean $\pm S D$. Analysis of variance was used to compare the mean values among three groups. Chi-square test and Fisher's exact test were used for the comparisons of fraction. Statistical significance was defined as $p<0.05$. All analyses were performed using the SPSS statistical package for Windows, ver. 12.0 (SPSS Inc, Chicago, IL, USA).

\section{Results}

There were no differences in the age of patients, duration of infertility, body mass index, the proportion of nullipara, AFC and basal endocrine profile among three groups (Table 1).

In group 1 and 3, no patient was lost to follow-up or withdrawn from the study and there were no cancellation of cycle by premature LH surge or any other reasons. In group 2, 1 out of 40 cycles initiated (2.5\%) was cancelled before ET, because no oocytes were obtained despite a follicular aspiration for oocyte retrieval. However, there was no significant difference in cycle cancellation rate among three groups (Table 2).

Total doses $(2,925.0 \pm 423.9$ IU vs. $2,905.0 \pm 421.8$ IU vs. 3,273.6 \pm

Table 1. Patients' characteristics

\begin{tabular}{lcccc}
\hline & $\begin{array}{c}\text { Group 1 } \\
\text { (MDP with } \\
\text { OCP) }\end{array}$ & $\begin{array}{c}\text { Group 2 } \\
\text { (MDP with- } \\
\text { out OCP) }\end{array}$ & $\begin{array}{c}\text { Group 3 } \\
\text { (GnRH ago- } \\
\text { nist low- } \\
\text { dose LP) }\end{array}$ & p-value \\
\hline No. of patients & 40 & 40 & 40 & \\
Age of patients (yr) & $36.7 \pm 3.1$ & $35.9 \pm 2.8$ & $36.4 \pm 3.3$ & $\mathrm{NS}^{\mathrm{a}}$ \\
Duration of infertility (mo) & $50.1 \pm 20.4$ & $46.8 \pm 18.8$ & $51.3 \pm 22.5$ & $\mathrm{NS}^{\mathrm{a}}$ \\
Body mass index (kg/m²) & $22.1 \pm 2.4$ & $22.1 \pm 2.1$ & $22.3 \pm 2.5$ & $\mathrm{NS}^{\mathrm{a}}$ \\
No. of nullipara & $19(47.5)$ & $21(52.5)$ & $22(55.0)$ & $\mathrm{NS}^{\mathrm{b}}$ \\
Antral follicle count & $4.6 \pm 1.5$ & $4.7 \pm 1.6$ & $4.7 \pm 1.7$ & $\mathrm{NS}^{\mathrm{a}}$ \\
Basal serum FSH (IU/L) & $8.8 \pm 1.2$ & $8.6 \pm 1.0$ & $8.8 \pm 1.2$ & $\mathrm{NS}^{\mathrm{a}}$ \\
Basal serum LH (IU/L) & $5.0 \pm 1.4$ & $5.3 \pm 1.2$ & $5.2 \pm 1.3$ & $\mathrm{NS}^{\mathrm{a}}$ \\
Basal serum E2 $(\mathrm{pg} / \mathrm{mL})$ & $48.3 \pm 9.6$ & $46.5 \pm 8.6$ & $47.2 \pm 9.3$ & $\mathrm{NS}^{\mathrm{a}}$ \\
Serum PRL $(\mathrm{ng} / \mathrm{mL})$ & $13.1 \pm 2.2$ & $14.1 \pm 2.7$ & $13.5 \pm 2.3$ & $\mathrm{NS}^{\mathrm{a}}$ \\
Serum T $(\mathrm{ng} / \mathrm{mL})$ & $0.3 \pm 0.2$ & $0.3 \pm 0.2$ & $0.2 \pm 0.3$ & $\mathrm{NS}^{\mathrm{a}}$ \\
Serum AMH $(\mathrm{ng} / \mathrm{mL})$ & $1.5 \pm 0.5$ & $1.6 \pm 0.5$ & $1.4 \pm 0.6$ & $\mathrm{NS}^{\mathrm{a}}$ \\
\hline
\end{tabular}

Values are presented as mean \pm SD or number (\%).

MDP, multiple-dose protocol; OCP, oral contraceptive pill; LP, long protocol; AMH, antimüllerian hormone; NS, not significant.

${ }^{\mathrm{a}}$ Analysis of variance; ${ }^{\mathrm{b}} \mathrm{Chi}$-square test.
$438.3 \mathrm{IU}$ ) and days (9.9 \pm 1.3 days vs. $9.8 \pm 1.3$ days vs. $11.3 \pm 1.5$ days) of $\mathrm{rhFSH}$ required for $\mathrm{COS}$ were significantly higher in group 3 than in group 1 or $2(p<0.001, p<0.001$ ) (Table 2). The number of follicles $\geq$ $14 \mathrm{~mm}$ and endometrial thickness on hCG day, number of oocytes retrieved were similar among three groups, but the number of mature oocytes ( $4.0 \pm 1.6$ vs. $2.8 \pm 1.2$ vs. $3.8 \pm 1.6$ ), fertilized oocytes (3.9 \pm 1.6 vs. $2.8 \pm 1.1$ vs. $3.8 \pm 1.6)$ and grade I, II embryos ( $2.2 \pm 1.1$ vs. 1.3 \pm 0.9 vs. $2.1 \pm 1.0$ ) were significantly lower in group 2 than in group 1 or 3 ( $p=0.001, p=0.002, p<0.001$, respectively) (Table 2). However, significant differences were not found among three groups regarding clinical pregnancy rate per cycle initiated, implantation rate and live birth rate per cycle initiated (Table 2).

\section{Discussion}

The present study evaluated the effectiveness of $\mathrm{GnRH}$ antagonist MDP with or without OCP pretreatment, compared with GnRH agonist low-dose LP that have been popularly used since the 1990's in poor responders undergoing IVF/ICSI. Total dose and duration of rhFSH used for COS were significantly higher in GnRH agonist low-dose LP group than in GnRH antagonist MDP groups, which suggests the advantage of $\mathrm{GnRH}$ antagonist protocol. It is well known advantage of GnRH antagonist protocols compared with GnRH agonist LP, and Howles et al. [18] reported that the GnRH antagonists offer comparable therapeutic efficacy to agonists and have a number of potential advantages over agonists for use in COS protocols, such as avoiding the initial "flare-up" of $\mathrm{LH}$, shortening the overall treatment period and reducing the risk of OHSS and menopausal side effects in women of advanced maternal age. In group 1, the numbers of mature oocytes, fertilized oocytes and grade I, II embryos were comparable to those in group 3, whereas these were significantly lower in group 2. This implicates OCP pretreatment in GnRH antagonist MDP is effective in improving the ovarian response to COS and IVF outcome with similar level to GnRH agonist low-dose LP. In patients with elevated basal serum FSH levels by diminished ovarian reserve, antral follicle sizes during the early follicular phase are often markedly heterogeneous because FSH-sensitive follicles are early exposed to gradient FSH concentrations during the preceding luteal phase. Therefore, in poor responders, ovarian stimulation without pituitary suppression is likely to induce asynchronous follicular development and a limited number of dominant follicles. The COS results of present study may result from pituitary suppression prior to ovarian stimulation. Actually, a study by Fanchin et al. [19] showed that luteal FSH suppression by either estradiol or $\mathrm{GnRH}$ antagonists administration improves the homogeneity of early antral follicles during the early follicular phase, thereby improving ovarian response to short GnRH agonist and antagonist protocols. 
Table 2. Comparison of controlled ovarian stimulation results and IVF/ICSI outcome

\begin{tabular}{|c|c|c|c|c|}
\hline & $\begin{array}{c}\text { Group } 1 \\
\text { (MDP with OCP) }\end{array}$ & $\begin{array}{c}\text { Group } 2 \\
\text { (MDP without OCP) }\end{array}$ & $\begin{array}{c}\text { Group } 3 \\
\text { (GnRH agonist low-dose LP) }\end{array}$ & $p$-value \\
\hline No. of cycles initiated & 40 & 40 & 40 & \\
\hline No. of cycles retrieved & 40 & 40 & 40 & \\
\hline No. of ET cycles & 40 & 39 & 40 & \\
\hline No. of cycles cancelled & 0 & $1(2.5)$ & 0 & $\mathrm{NS}^{\mathrm{a}}$ \\
\hline Total dose of rhFSH (IU) & $2,925.0 \pm 423.9$ & $2,905.0 \pm 421.8$ & $3,273.6 \pm 438.3$ & $<0.001^{b}$ \\
\hline Days of rhFSH injected & $9.9 \pm 1.3$ & $9.8 \pm 1.3$ & $11.3 \pm 1.5$ & $<0.001^{b}$ \\
\hline \multicolumn{5}{|l|}{ On the day of $\mathrm{hCG}$ injection } \\
\hline No. of follicles $\geq 14$ mm & $4.8 \pm 1.8$ & $4.3 \pm 1.3$ & $4.5 \pm 1.4$ & $N S^{b}$ \\
\hline EMT $(\mathrm{mm})$ & $10.2 \pm 1.7$ & $10.4 \pm 1.7$ & $10.5 \pm 1.9$ & $N S^{b}$ \\
\hline No. of cycle with premature LH surge & 0 & 0 & 0 & $\mathrm{NS}^{\mathrm{a}}$ \\
\hline No. of cycles with ICSI & $19(47.5)$ & $16(40.0)$ & $18(45.0)$ & $N S^{a}$ \\
\hline No. of oocytes retrieved & $4.9 \pm 2.0$ & $4.2 \pm 1.7$ & $4.8 \pm 1.9$ & $\mathrm{NS}^{\mathrm{b}}$ \\
\hline No. of mature oocytes & $4.0 \pm 1.6$ & $2.8 \pm 1.2$ & $3.8 \pm 1.6$ & $0.001^{b}$ \\
\hline No. of fertilized oocytes & $3.9 \pm 1.6$ & $2.8 \pm 1.1$ & $3.8 \pm 1.6$ & $0.002^{b}$ \\
\hline No. of grade I, II embryos & $2.2 \pm 1.1$ & $1.3 \pm 0.9$ & $2.1 \pm 1.0$ & $<0.001^{b}$ \\
\hline No. of embryos frozen & $0.3 \pm 0.6$ & $0.1 \pm 0.2$ & $0.3 \pm 0.5$ & $N S^{b}$ \\
\hline No. of embryos transferred & $2.9 \pm 0.2$ & $2.8 \pm 0.2$ & $2.9 \pm 0.3$ & $N S^{b}$ \\
\hline Clinical PR per cycle initiated (\%) & $37.5(14 / 40)$ & $22.5(9 / 40)$ & $32.5(13 / 40)$ & $N S^{\mathrm{a}}$ \\
\hline Implantation rate (\%) & $15.5(18 / 116)$ & $9.6(11 / 114)$ & $13.7(16 / 117)$ & $\mathrm{NS}^{\mathrm{a}}$ \\
\hline Miscarriage rate per clinical pregnancy (\%) & $14.3(2 / 14)$ & $11.1(1 / 9)$ & $15.4(2 / 13)$ & $N S^{a}$ \\
\hline Live birth rate per cycle initiated (\%) & $30.0(12 / 40)$ & $20.0(8 / 40)$ & $27.5(11 / 40)$ & $\mathrm{NS}^{\mathrm{a}}$ \\
\hline Twin PR per clinical pregnancy (\%) & $28.6(4 / 14)$ & $20.0(2 / 10)$ & $23.1(3 / 13)$ & $\mathrm{NS}^{\mathrm{a}}$ \\
\hline
\end{tabular}

Values are presented as mean \pm SD or number $(\%)$.

MDP, multiple-dose protocol; OCP, oral contraceptive pill; LP, long protocol; ET, embryo transfer; EMT, endometrial thickness; PR, pregnancy rate; NS, not significant.

${ }^{\mathrm{a}}$ Chi-square test or Fisher's exact test; ${ }^{\mathrm{b}}$ Analysis of variance.

In terms of clinical pregnancy rate in our study, GnRH antagonist MDP with OCP pretreatment seemed to work better than GnRH antagonist MDP without OCP pretreatment, but the difference was not statistically significant. GnRH antagonist MDP with OCP pretreatment yielded comparable IVF results and pregnancy outcome to $\mathrm{GnRH}$ agonist low-dose LP with fewer dose and days of rhFSH required.

Assessment of various $\mathrm{COS}$ regimens for poor responders is difficult because there is no universal definition for poor responder and lack of a large number of prospective randomized trials demonstrating the advantage of a specific single protocol [20]. However, low-dose $\mathrm{GnRH}$ agonist protocols, microdose $\mathrm{GnRH}$ agonist flare regimens and $\mathrm{GnRH}$ antagonist protocols are considered as beneficial regimens in poor responders $[1,20]$. Olivennes et al. [4] prospectively analyzed 98 patients with low-dose GnRH agonist protocol, which leuprolide acetate of $0.5 \mathrm{mg}$ daily was initiated in the mid-luteal phase and then decreased to $0.25 \mathrm{mg}$ daily after menses when gonadotropin stimulation was commenced. They reported improvement of COS results with reduced duration and doses of gonadotropin stimulation. Several studies have recently evaluated GnRH antagonist protocols comparing to alternative regimens designed for poor responders. Copperman suggested $\mathrm{GnRH}$ antagonist protocol in poor responders could reduce the cancellation rate of the cycle and increase the ongoing pregnancy rate, when compared with GnRH agonist microdose flare protocol [21]. Cheung et al. [22] also reported a fixed, multiple-dose GnRH antagonist protocol could be utilized for poor responders although they failed to demonstrate an overall improvement in ovarian responsiveness. These recent studies implicate the effectiveness of $\mathrm{GnRH}$ antagonist use in $\mathrm{COS}$ for poor responders.

The OCP pretreatment in IVF cycles is the simplest method for cycle scheduling, and it can be beneficial in improving ovarian responses by inhibition of intrinsic gonadotropins before ovarian stimulation. A recent meta-analysis demonstrated no significant differences between patients with and those without OCP pretreatment regarding the number of cumulus-oocyte complexes, fertilization rates and ongoing pregnancy rates [14]. However, we showed OCP pretreatment in GnRH antagonist cycles can offer advantages in IVF results in poor responders. Copperman also reported a higher proportion of patients obtained more than eight oocytes, a significantly higher pregnancy rate when OCP pretreatment was performed in poor responders [21]. It is thought that OCP pretreatment in GnRH antagonist cycles can result in different ovarian response to $\operatorname{COS}$ and IVF results in poor responders, compared with normal responders, and OCP pretreatment 
might be more advantageous in poor responders than in normal responders.

The present study demonstrated that GnRH antagonist MDP with OCP pretreatment is at least as effective as GnRH agonist low-dose $\mathrm{LP}$ in poor responders and can be advantageous to poor responders because of the shortened time required for follicular maturation and the diminished amount of rhFSH required to provide adequate ovarian stimulation. Therefore, $\mathrm{GnRH}$ antagonist protocol with OCP pretreatment might be a useful choice of COS for poor responders. Larger studies with standardized methods will be needed for confirmation of our conclusions.

\section{Conflict of interest}

No potential conflict of interest relevant to this article was reported.

\section{References}

1. Surrey ES. Management of the poor responder: the role of $\mathrm{GnRH}$ agonists and antagonists. J Assist Reprod Genet 2007;24:613-9.

2. Porter RN, Smith W, Craft IL, Abdulwahid NA, Jacobs HS. Induction of ovulation for in-vitro fertilisation using buserelin and gonadotropins. Lancet 1984;2:1284-5.

3. Feldberg D, Farhi J, Ashkenazi J, Dicker D, Shalev J, Ben-Rafael Z. Minidose gonadotropin-releasing hormone agonist is the treatment of choice in poor responders with high follicle-stimulating hormone levels. Fertil Steril 1994;62:343-6.

4. Olivennes F, Righini C, Fanchin R, Torrisi C, Hazout A, Glissant M, et al. A protocol using a low dose of gonadotrophin-releasing hormone agonist might be the best protocol for patients with high follicle-stimulating hormone concentrations on day 3. Hum Reprod 1996;11:1169-72.

5. De Placido G, Mollo A, Clarizia R, Strina I, Conforti S, Alviggi C. Gonadotropin-releasing hormone $(\mathrm{GnRH})$ antagonist plus recombinant luteinizing hormone vs. a standard $\mathrm{GnRH}$ agonist short protocol in patients at risk for poor ovarian response. Fertil Steril 2006;85:247-50.

6. Mahutte NG, Arici A. Role of gonadotropin-releasing hormone antagonists in poor responders. Fertil Steril 2007;87:241-9.

7. Schoolcraft WB, Surrey ES, Minjarez DA, Stevens JM, Gardner DK. Management of poor responders: can outcomes be improved with a novel gonadotropin-releasing hormone antagonist/letrozole protocol? Fertil Steril 2008;89:151-6.

8. Fisch JD, Keskintepe L, Sher G. Gonadotropin-releasing hormone agonist/antagonist conversion with estrogen priming in low responders with prior in vitro fertilization failure. Fertil Steril 2008; 89:342-7.
9. Lainas TG, Sfontouris IA, Papanikolaou EG, Zorzovilis JZ, Petsas GK, Lainas GT, et al. Flexible GnRH antagonist versus flare-up GnRH agonist protocol in poor responders treated by IVF: a randomized controlled trial. Hum Reprod 2008;23:1355-8.

10. Griesinger G, Diedrich K, Tarlatzis BC, Kolibianakis EM. GnRH-antagonists in ovarian stimulation for IVF in patients with poor response to gonadotrophins, polycystic ovary syndrome, and risk of ovarian hyperstimulation: a meta-analysis. Reprod Biomed Online 2006;13:628-38.

11. Meldrum D, Scott R, Levy MJ, Alper M, Noyes N. A pilot study to assess oral contraceptive (OC) pretreatment in women undergoing controlled ovarian hyperstimulation $(\mathrm{COH})$ in ganirelix acetate cycles. Fertil Steril 2002;78:S176.

12. Barmat LI, Chantilis SJ, Hurst BS, Dickey RP. A randomized prospective trial comparing gonadotropin-releasing hormone $(\mathrm{GnRH})$ antagonist/recombinant follicle-stimulating hormone ( $\mathrm{rFSH}$ ) versus $\mathrm{GnRH}$-agonist/rFSH in women pretreated with oral contraceptives before in vitro fertilization. Fertil Steril 2005;83:321-30.

13. Kolibianakis EM, Papanikolaou EG, Camus $M$, Tournaye $H$, Van Steirteghem AC, Devroey P. Effect of oral contraceptive pill pretreatment on ongoing pregnancy rates in patients stimulated with GnRH antagonists and recombinant FSH for IVF. A randomized controlled trial. Hum Reprod 2006;21:352-7.

14. Griesinger G, Venetis CA, Marx T, Diedrich K, Tarlatzis BC, Kolibianakis EM. Oral contraceptive pill pretreatment in ovarian stimulation with GnRH antagonists for IVF: a systematic review and meta-analysis. Fertil Steril 2008;90:1055-63.

15. Kim $\mathrm{CH}, \mathrm{Cho}$ YK, Mok JE. The clinical usefulness of basal follicle stimulating hormone levels measured by immunoradiomeric assay (IRMA) as a prognostic indicator of ovarian reserve. Korean J Obstet Gynecol 1995;38:1924-36.

16. Bancsi LF, Broekmans FJ, Eijkemans MJ, de Jong FH, Habbema JD, te Velde ER. Predictors of poor ovarian response in in vitro fertilization: a prospective study comparing basal markers of ovarian reserve. Fertil Steril 2002;77:328-36.

17. Bancsi LF, Broekmans FJ, Looman CW, Habbema JD, te Velde ER. Impact of repeated antral follicle counts on the prediction of poor ovarian response in women undergoing in vitro fertilization. Fertil Steril 2004;81:35-41.

18. Howles CM, Kim CH, Elder K. Treatment strategies in assisted reproduction for women of advanced maternal age. Int Surg 2006; 91:S37-54.

19. Fanchin R, Mendez Lozano DH, Schonauer LM, Cunha-Filho JS, Frydman R. Hormonal manipulations in the luteal phase to coordinate subsequent antral follicle growth during ovarian stimulation. Reprod Biomed Online 2005;10:721-8.

20. Loutradis D, Vomvolaki E, Drakakis P. Poor responder protocols 
for in-vitro fertilization: options and results. Curr Opin Obstet Gynecol 2008;20:374-8.

21. Copperman AB. Antagonists in poor-responder patients. Fertil Steril 2003;80 Suppl 1:S16-24.
22. Cheung LP, Lam PM, Lok IH, Chiu TT, Yeung SY, Tjer CC, et al. GnRH antagonist versus long $\mathrm{GnRH}$ agonist protocol in poor responders undergoing IVF: a randomized controlled trial. Hum Reprod 2005;20:616-21. 